\title{
BMJ Open Review of novel therapeutics in cardiac arrest (ReNTICA): systematic review protocol
}

\author{
Travis W Murphy (D) , ${ }^{1}$ Garrett Snipes (D) ,2 Muhammad Abdul Baker Chowdhury, ${ }^{3}$ \\ Patti McCall-Wright, ${ }^{4}$ Elizabeth Aleong, ${ }^{5}$ Noelle Taylor, ${ }^{5}$ Maiya-Mari Messina, ${ }^{5}$ \\ Gabriela Carrazana, ${ }^{5}$ Carolina B Maciel, ${ }^{6}$ Torben $\mathrm{K}$ Becker ${ }^{3}$
}

To cite: Murphy TW, Snipes G, Chowdhury MAB, et al. Review of novel therapeutics in cardiac arrest (ReNTICA): systematic review protocol. BMJ Open 2022;12:e053304. doi:10.1136/ bmjopen-2021-053304

- Prepublication history and additional supplemental material for this paper are available online. To view these files, please visit the journal online (http://dx.doi.org/10.1136/ bmjopen-2021-053304).

Received 11 May 2021 Accepted 08 December 2021

Check for updates

(c) Author(s) (or their employer(s)) 2022. Re-use permitted under CC BY-NC. No commercial re-use. See rights and permissions. Published by BMJ.

${ }^{1}$ Department of Emergency Medicine, University of Florida, Gainesville, Florida, USA

${ }^{2}$ Internal Medicine, University of Florida College of Medicine, Gainesville, Florida, USA

${ }^{3}$ Emergency Medicine, University of Florida Health, Gainesville,

Florida, USA

${ }^{4}$ Health Science Center Libraries, University of Florida Clinical and Translational Science Institute (CTSI), Gainesville, Florida, USA ${ }^{5}$ University of Florida, Gainesville, Florida, USA

${ }^{6}$ Neurology and Neurosurgery, University of Florida Health, Gainesville, Florida, USA

Correspondence to Dr Travis W Murphy; twm51@miami.edu

\section{ABSTRACT}

Introduction Cardiac arrest remains a common and devastating cause of death and disability worldwide. While targeted temperature management has become standard of care to improve functional neurologic outcome, few pharmacologic interventions have shown similar promise. Methods/analysis This systematic review will focus on prospective human studies from 2015 to 2020 available in PubMed, Web of Science and EMBASE with a primary focus on impact on functional neurologic outcome. Prospective studies that include pharmacologic agents given during or after cardiac arrest will be included. Study selection will be in keeping with Preferred Reporting Items for Systematic Reviews and Meta-Analyses guidelines. If sufficient data involving a given agent are available, a meta-analysis will be conducted and compared with current evidence for therapies recommended in international practice guidelines.

Ethics and dissemination Formal ethical approval will not be required as primary data will not be collected. The results will be disseminated through peer-reviewed publication, conference presentation and lay press. PROSPERO registration number International Prospective Register for Systematic Reviews (CRD42021230216).

\section{INTRODUCTION}

Cardiac arrest remains a common and often devastating cause of death and disability worldwide. ${ }^{1}$ Outcomes are contingent on the severity of overall hypoxic-ischaemic brain injury burden, which comprises primary injury during circulation standstill and ongoing secondary brain injury that occurs in the aftermath of resuscitation. Aside from targeted temperature management, therapeutic options targeting improvement in neurologic outcome are scarce and recent data have cast doubt on even this guidelinerecommended therapy. ${ }^{2} 3$ International practice guidelines currently recommend epinephrine, amiodarone and lidocaine/ lignocaine. ${ }^{4-6}$ While there is good evidence for improved survival with these medications, there is little evidence for a positive impact
Strengths and limitations of this study

- Systematic review and meta-analysis of prospective trials investigating therapeutic agents in cardiac arrest.

- Inclusion of outcome measurements to quantify the effect of proposed therapeutic interventions on neurologic function after cardiac arrest.

- Limitation in the number of studies investigating a given therapy may diminish observed effects.

- Potential identification of therapies with positive impact on functional outcome following cardiac arrest.

of these medications on functional neurologic outcomes specifically. ${ }^{4} 67$ Functional neurologic outcome is less commonly the primary focus of cardiac arrest research but arguably a more granular patient-centred variable rather than rate of return of spontaneous circulation or survival. Unlike targeted temperature management, few advances have been made in pharmacologic approaches to improve functionally intact neurologic survival following cardiac arrest. ${ }^{2689}$ Several novel compounds have been investigated in animal studies, with some notation of effect on neurologic function, though only a small minority have made the transition from animal models into human trials. ${ }^{8}$ The review proposed here will focus on identifying the best available data from human studies and report on therapies that may not have been explicitly mentioned in international guidelines to date.

A similar review of the literature was performed in 2015, though this was focused on cataloguing the therapies used and did not focus on studies that included functional outcome measurements. ${ }^{9}$ Another recent systematic review reported the rate of translation from animal models to human trials for therapies targeted at cardiac arrest. ${ }^{8}$ Though again, this review did not have a specific focus 
on functional neurologic outcome. While the review published by Lind et al identified the large number of experimental therapies targeted at postcardiac arrest physiology, the authors noted a relative dearth of clinical trials investigating those same therapies in humans. ${ }^{8}$ Additionally, the review published in 2021 did not compare the effects of different pharmacologic agents. ${ }^{8}$ The review proposed here seeks to compile the best available evidence for pharmacologic interventions that will improve functional neurologic outcomes in humans following cardiac arrest and compare this directly to current practice guidelines.

\section{Objectives}

The objective of our study is to systematically review the literature for prospective studies that evaluated the performance of pharmacologic agents used in adult cardiac arrest compared with standard resuscitation treatments currently advocated in practice guidelines. This will include studies regardless of initial cardiac rhythm and independent of the use of targeted temperature management.

\section{METHODS AND DESIGN Population}

The systematic review will focus on studies that include patients aged $>15$ years who have been resuscitated from cardiac arrest but are not conscious on return of spontaneous circulation with arms for both intervention and control.

\section{Interventions}

The interventions to be evaluated include any pharmacologic agent given during cardiac arrest itself or in the immediate postarrest period (defined as the initial 24 hours).

\section{Comparisons}

The added benefit of the interventions identified will be compared with current international practice guidelines and the pharmacologic agents advocated there (epinephrine, amiodarone and lidocaine/lignocaine).

\section{Outcome}

The primary outcomes required of included studies will be survival and neurologic function as defined by one of the following neurologic scales: Cerebral Performance Category, modified Rankin Scale and Glasgow Outcome Scale/Glasgow Outcome Scale-Extended.

\section{Study design}

The systematic review and meta-analysis will include prospective therapeutic studies investigating the use of a pharmacologic agent during or after cardiac arrest with a primary focus on effects of neurologic outcome. This will include systematic reviews, meta-analyses, randomised control trials, adaptive clinical trials, prospective cohort and observational studies and non-randomised clinical trials. Studies that compare one intervention to standard resuscitation as the control will be included. No minimum number of included subjects will be required. The review will exclude studies without a control group using either placebo or current standard care. Retrospective studies will be excluded including retrospective cohorts, casecontrol studies, cross-sectional studies, case reports and case series.

\section{Search strategy}

A three-step process will be used to identify eligible studies, including an initial search, title and abstract screening and full-text manuscript review. A professional systematic review librarian (PMW) will develop search criteria in discussion with the authors to include all relevant studies pertaining to adult, human studies of pharmacologic treatment of cardiac arrest. The databases that will be searched are PubMed, Web of Science and EMBASE from the year 2015 to 2020, inclusive. No language restrictions will be applied. Figure 1 shows an example search algorithm for PubMed. Initial deduplication will be performed using EndNote (Clarivate Analytics, Philadelphia, Pennsylvania, USA). ${ }^{10}$

\section{Study selection}

Literature search results will be uploaded from EndNote and screened through DistillerSR (Evidence Partners, Ottawa, Ontario, Canada). Study titles and abstracts will be screened for relevance in duplicate, blindly and independently, by four reviewers (EA, NT, GC, M-MM) and adjudicated by a senior author (TWM, CBM). Eligible studies will then be assessed again for inclusion and for quality in secondary screening through review of full-text manuscripts before data abstraction. This process will be reported using the Preferred Reporting Items for Systematic Reviews and Meta-Analyses (PRISMA) flow diagram. The PRISMA-P Checklist pertaining to this protocol is available as online supplemental file 1. Any conflicting remarks regarding studies will be adjudicated through discussion before inclusion in the final analysis.

\section{Quality assessment}

Each article will undergo initial screening in parallel by two independent reviewers to minimise bias. All selected articles will be reviewed with senior authors during fulltext review. Cochrane tools for assessment of study quality will be used as appropriate (ROBINS-1 and RoB 2.0). Two independent authors will assess the risks of bias in studies considered for full-text review in order to determine feasibility of a meta-analysis. Conflicts will be adjudicated with discussion and involvement of a third author (TWM or $\mathrm{CBM}$ ) as necessary.

\section{Data extraction}

Quantitative data will be extracted from studies meeting inclusion on full-text review by a professional biostatistician $(\mathrm{MABC})$. Data extracted will be specifically those pertinent to the systematic review and all others that fit into the synthesis of outcome parameters from all 
Cardiac Arrest

cardiac arrest[MeSH Terms] OR arrest, cardiopulmonary[MeSH Terms] OR heart arrest[MeSH Terms] OR deep hypothermic circulatory arrest[MeSH Terms] OR ventricular fibrillation [MeSH Terms] OR ventricular tachycardia[MeSH Terms] OR asystole[MeSH Terms] OR cardiopulmonary resuscitation[MeSH Terms] OR advanced cardiac life support[MeSH Terms] OR cardiac death[MeSH Terms] OR sudden cardiac death [MeSH Terms] OR cardiac death, sudden [MeSH Terms] OR cardiac arrest[Text Word] OR cardiopulmonary arrest[Text Word] OR heart arrest[Text Word] OR circulatory arrest[Text Word] OR ventricular fibrillation[Text Word] OR ventricular tachycardia[Text Word] OR pulseless electrical activity[Text Word] OR asystole[Text Word] OR cardiovascular arrest[Text Word] OR car diopulmonary arrest[Text Word] OR cardiopulmonary resuscitation[Text Word] OR defibrillation[Text Word] OR advanced cardiac life support[Text Word] OR ACLS[Text Word] OR cardiac death[Text Word] OR sudden cardiac death[Text Word] OR fatal arrhythmia[Text Word] 2015:2021[pdat]

Results 1: 57,400

AND

Survival

survival[MeSH Terms] OR mortality[MeSH Terms] OR survival[Text Word] OR mortality[Text Word] 2015:2021[pdat]

Results 2: 705, 713

AND

Functional Outcome

glasgow outcome scale[MeSH Terms] OR glasgow outcome scale[Text Word] OR modified rankin scale[Text Word] OR cerebral performance categories score[Text Word] OR prognosis[MeSH Terms] OR disability evaluation[MeSH Terms] OR prognosis[Text Word] OR disability evaluation[Text Word] 2015:2021[pdat]

Results 3: 603,078

AND

\section{Pharmacology}

drug*[Text Word] OR drug therapy[Text Word] OR medication[Text Word] OR phamacolog*[Text Word] OR pharmaceutic*[Text Word] OR therap*[Text Word] OR injection[Text Word] OR infusion [Text Word] OR cardiovascular agents[Text Word] OR drug administration schedule[Text Word] OR therapeutics[Text Word] OR in travenous administration[Text Word] OR dose response relationship [Text Word] OR cardiovascular agents[MeSH Terms] OR therapeutics[MeSH Terms] OR drug administration schedule[MeSH Terms] OR dose response relationship, drug[MeSH Terms] OR vasopressor agents[MeSH Terms] OR epinephrine[MeSH Terms] OR vasopressins[MeSH Terms] OR norepinephrine[MeSH Terms] OR Simendan[MeSH Terms] OR methoxamine[MeSH Terms] OR amiodarone[MeSH Terms] OR sodium channel blockers[MeSH Terms] OR lidocaine[MeSH Terms] OR lignocaine[MeSH Terms] OR procainamide[MeSH Terms] OR flecainide[MeSH Terms] OR mexiletine[MeSH Terms] OR quinidine[MeSH Terms] OR adrenergic beta blockers[MeSH Terms] OR sotalol[MeSH Terms] OR apo labetalol[MeSH Terms] OR metoprolol[MeSH Terms] OR nadolol[MeSH Terms] OR bisoprolol[MeSH Terms] OR carvedilol[MeSH Terms] OR isoproterenol[MeSH Terms] OR calcium channel blockers[MeSH Terms] OR diltiazem[MeSH Terms] OR verapamil[MeSH Terms] OR digoxin[MeSH Terms] OR isoprenaline[MeSH Terms] OR theophylline[MeSH Terms] OR aminophylline[MeSH Terms] OR magnesium[MeSH Terms] OR thrombolytic agents[MeSH Terms] OR thrombolytic drugs[MeSH Terms] OR thrombolytic therapies[MeSH Terms] OR corticosteroids [MeSH Terms] OR sodium bicarbonate[MeSH Terms] OR erythropoietin[MeSH Terms] OR crystalloid solutions [MeSH Terms] OR ascorbic acid[MeSH Terms] OR vitamin e[MeSH Terms] OR vitamin b1[MeSH Terms] OR melatonin[MeSH Terms] OR colloids[MeSH Terms] OR xenon[MeSH Terms] OR argon[MeSH Terms] OR hydrogen [MeSH Terms] OR hydrogen sulfide[MeSH Terms] OR nitric oxide[MeSH Terms] OR carbon monoxide[MeSH Terms] OR anesthetic agents[MeSH Terms] OR anesthetic drugs[MeSH Terms] OR propofol[MeSH Terms] OR ketamine[MeSH Terms] OR vasopressor[Text Word] OR epinephrine[Text Word] OR vasopressin[Text Word] OR norepinephrine[Text Word] OR levosimendan[Text Word] OR simendan[Text Word] OR methoxamine[Text Word] OR amiodarone[Text Word] OR sodium channel blockers[Text Word] OR lidocaine[Text Word] OR lignocaine[Text Word] OR procainamide[Text Word] OR flecainide[Text Word] OR mexiletine[Text Word] OR quinidine[Text Word] OR beta blocker[Text Word] OR sotalol[Text Word] OR labetaloI[Text Word] OR metoprolol[Text Word] OR nadolol[Text Word] OR bisoprolol[Text Word] OR carvediloI[Text Word] OR isoproterenol[Text Word] OR calcium channel blocker[Text Word] OR diltiazem[Text Word] OR verapamil[Text Word] OR at ropine[Text Word] OR atropine[MeSH Terms] OR digoxin[Text Word] OR isoprenaline[Text Word] OR theopylline[Text Word] OR magnesium[Text Word] OR thrombolytic[Text Word] OR corticosteroids[Text Word] OR sodium bicarbonate[Text Word] OR erythropoietin[Text Word] OR crystalloid[Text Word] OR colloid[Text Word] OR vitamin c[Text Word] OR ascorbic acid[Text Word] OR vitamin e[Text Word] OR vitamin b[Text Word] OR thiamine[Text Word] OR edavarone[Text Word] OR melatonin[Text Word] OR medical gas[Text Word] OR xenon[Text Word] OR argon[Text Word] OR hydrogen[Text Word] OR hydrogen sulfide[Text Word] OR nitric oxide[Text Word] OR carbon monoxide[Text Word] OR anesthetics[Text Word] OR propofol[Text Word] OR ketamine[Text Word] OR adrenergic alpha agonists[MeSH Terms] OR Adrenergic alpha-1 Receptor Antagonists[MeSH Terms] OR adrenergic beta antagonists[MeSH Terms] OR adjuvants, anesthesia[MeSH Terms] OR analgesics[MeSH Terms] OR anesthetics, dissociative[MeSH Terms] OR anesthetics, local[MeSH Terms] OR anti arrhythmia agents[MeSH Terms] OR antihypertensive agents[MeSH Terms] OR antioxidants[MeSH Terms] OR bronchodilator agents[MeSH Terms] OR cardiotonic agents[MeSH Terms] OR cardiovascular agents[MeSH Terms] OR cytochrome $p 450$ CYP1A2 inhibitors[MeSH Terms] OR cytochrome p 450 CYP2C9 inhibitors[MeSH Terms] OR cytochrome p 450 CYP2D6 inhibitors[MeSH Terms] OR cytochrome p 450 CYP3A inhibitors[MeSH Terms] OR enzyme inhibitors[MeSH Ter ms] OR excitatory amino acid antagonists[MeSH Terms] OR fibrinolytic agents[MeSH Terms] OR muscarinic antagonists[MeSH Terms] OR mydriatics[MeSH Terms] OR parasympatholytics[MeSH Terms] OR phosphodiesterase 3 inhibitors[MeSH Terms] OR potassium channel blockers[MeSH Terms] OR purinergic p1 receptor antagonists[MeSH Terms] OR sympathomimetics[MeSH Terms] OR sympatholytics[MeSH Terms] OR vasoconstrictor agents[MeSH Terms] OR vasodilator agents[MeSH Terms] OR vitamins[MeSH Terms] OR Adrenergic alphaAgonists[Text Word] OR Adrenergic alpha-1 Receptor Antagonists[Text Word] OR Adrenergic beta-Antagonists[Text Word] OR Adjuvants, Anesthesia[Text Word] OR Analgesics[Text Word] OR Anesthetics, Dissociative[Text Word] OR Anesthetics, Local[Text Word] OR Anti-Arrhythmia Agents[Text Word] OR Antihypertensive Agents[Text Word] OR Antioxidants[Text Word] OR Bronchodilator Agents[Text Word] OR Cardiotonic Agents[Text Word] OR Cardiovascular Agents[Text Word] OR Cytochrome P-450 CYP1A2 Inhibitors[Text Word] OR Cytochrome P-450 CYP2C9 Inhibitors[Text Word] OR Cytochrome P-450 CYP2D6 Inhibitors[Text Word] OR Cytochrome P-450 CYP3A Inhibitors[Text Word] OR Enzyme Inhibitors[Text Word] OR Excitatory Amino Acid Antagonists[Text Word] OR Fibrinolytic Agents[Text Word] OR Muscarinic Antagonists[Text Word] OR Mydriatics[Text Word] OR Parasympatholytics[Text Word] OR Phosphodiesterase 3 Inhibitors[Text Word] OR Potassium Channel Blockers[Text Word] OR Purinergic P1 Receptor Antagonists[Text Word] OR Sympathomimetics[Text Word] OR Sympatholytics[Text Word] OR Vasoconstrictor agents[Text Word] OR Vasodilator 2015:2021[pdat]

Results 4: 2, 723, 077

Combined Results $1+2+3+4: 4308$

Date: December 7, 2020

Figure 1 Example search algorithm for PubMed. 
studies and meet the potential for inclusion in a metaanalysis. This will include demographics, characteristics of cardiac arrest, medications administered and outcome parameters as well as any data that are available across all included studies. Data extraction will be independently crosschecked by a senior author and discrepancies resolved through discussion with other senior authors. Data produced from this systematic review including the statistical code and dataset of articles screened will be published in a data repository.

\section{Endpoint}

Results of the systematic review will be grouped by drug and drug class. The primary outcomes will be survival and neurologic function as defined by one of the following performance scales: Cerebral Performance Category, modified Rankin Scale and Glasgow Outcome Scale/ Glasgow Outcome Scale-Extended. Provided they are available in the original studies, secondary outcomes will be Intensive Care Unit (ICU) length of stay, ventilator days, rates of sepsis, rates of pneumonia, rate of tracheostomy, rate of acute kidney injury and need for renal replacement therapy (in the hospital or afterwards). We will also include secondary outcomes of functional capacity including Barthel Index, Katz Index, LawtonBrody Instrumental Activities of Daily Living Scale and rates of discharge to rehab facility if included in the original studies. Any follow-up duration will be accepted as there is considerable variability in the existing literature.

\section{Patient and public involvement}

No patients were involved with the planning of this protocol.

\section{ANALYSIS}

\section{Descriptive analysis}

A narrative synthesis of the final studies included will be developed based on the different pharmacologic agents identified. The impact of each of these agents on the primary and secondary outcome mentioned above will be described in addition to a formal meta-analysis of studies using each pharmacologic agent identified.

\section{Statistical analysis}

The primary focus of this review is to detect evidence for the impact of the pharmacologic agents identified on survival and functional neurologic outcome. As functional outcome is reported with some degree of heterogeneity, we are including three of the most widely reported functional outcome scales and not limiting to one over the others. As functional neurologic outcome is not always explicitly reported using one of these scales, we do anticipate some limitations in the ability to directly compare one agent to another. However, when available, pharmacologic agents will be compared as equitably as possible using all available outcome parameters reported in the index studies. The percentage of patients receiving a given pharmacologic agent with a favourable neurologic outcome according to each specific scale will be reported. Based on the availability of data from primary sources, subgroup analysis within cohorts treated with the same pharmacologic agent will also be performed to identify populations most likely to benefit from a given agent.

\section{Data synthesis}

Results will be presented in accordance with the PRISMA statement. A PRISMA flow diagram will be used to summarise study selection. Tabulated data showing relative proportion of patients with favourable functional neurologic outcome for each pharmacologic agent will be presented. We will rank agents by proportion of favourable outcomes. For secondary outcome variables, we will present synthesised data as available in separate tables but will otherwise provide a separate narrative summary of the data available for each agent. We will produce a hierarchy of pharmacologic agents based on the quality of evidence available and degree of effects on outcome variables.

\section{Meta-analysis}

A meta-analysis of the pharmacologic agents found to have been studied with a focus on functional neurologic outcome will be performed. The results of this meta-analysis will then be compared with the best available evidence for medications recommended in practice guidelines to provide context and rank relative efficacy.

\section{DISCUSSION}

This systematic review and meta-analysis will provide evidence for further study or use of compounds that are most likely to benefit patients following cardiac arrest in terms of functional status. The conclusions will be the result of careful accumulation of the highest-quality evidence available and will compare to current practice guidelines to place the effects in context. With a primary focus on the ability of a given pharmacologic agent to not only provide a survival benefit but also to protect the neurologic function of patients following cardiac arrest, this review and meta-analysis will be unique in its aim to identify agents with the greatest potential to benefit these patients.

\section{ETHICS AND DISSEMINATION}

This study does not involve human participants. No ethical or safety considerations were considered based on the nature of this review. Dissemination of findings through a peer-reviewed publication upon the conclusion of the meta-analysis.

\section{Twitter Carolina B Maciel @neurologyrules}

Contributors TWM: Conception and design of work, analysis, drafting of work and final approval. GS: Conception, design and drafting of work. MABC, PM-W and CBM: Conception and design of work and analysis. EA, NT, M-MM and GC: Analysis. TKB: Conception and design of work. All authors have reviewed and contributed to this final written manuscript. 
Funding This project received funding from the University of Florida Center for Translational Science Institute (Voucher \#LSR06-001) to support professional library services.

Competing interests None declared.

Patient consent for publication Not applicable.

Provenance and peer review Not commissioned; externally peer reviewed.

Supplemental material This content has been supplied by the author(s). It has not been vetted by BMJ Publishing Group Limited (BMJ) and may not have been peer-reviewed. Any opinions or recommendations discussed are solely those of the author(s) and are not endorsed by BMJ. BMJ disclaims all liability and responsibility arising from any reliance placed on the content. Where the content includes any translated material, BMJ does not warrant the accuracy and reliability of the translations (including but not limited to local regulations, clinical guidelines, terminology, drug names and drug dosages), and is not responsible for any error and/or omissions arising from translation and adaptation or otherwise.

Open access This is an open access article distributed in accordance with the Creative Commons Attribution Non Commercial (CC BY-NC 4.0) license, which permits others to distribute, remix, adapt, build upon this work non-commercially, and license their derivative works on different terms, provided the original work is properly cited, appropriate credit is given, any changes made indicated, and the use is non-commercial. See: http://creativecommons.org/licenses/by-nc/4.0/.

\section{ORCID iDs}

Travis W Murphy http://orcid.org/0000-0001-8985-7518

Garrett Snipes http://orcid.org/0000-0002-2078-8574

\section{REFERENCES}

1 Kiguchi T, Okubo M, Nishiyama C, et al. Out-Of-Hospital cardiac arrest across the world: first report from the International liaison Committee on resuscitation (ILCOR). Resuscitation 2020;152:39-49.
2 Panchal AR, Berg KM, Hirsch KG. American heart association focused update on advanced cardiovascular life support: use of advanced airways, vasopressors, and extracorporeal cardiopulmonary resuscitation during cardiac arrest: an update to the American heart association guidelines F. Circulation 2019;2019:E881-94.

3 Dankiewicz J, Cronberg T, Lilja G, et al. Hypothermia versus normothermia after out-of-hospital cardiac arrest. N Engl J Med 2021;384:2283-94.

4 Panchal AR, Berg KM, Kudenchuk PJ. American heart association focused update on advanced cardiovascular life support use of antiarrhythmic drugs during and immediately after cardiac arrest: an update to the American heart association guidelines for cardiopulmonary resuscitation and em. Circulation 2018;2018: $740-9$

5 Holmberg MJ, Issa MS, Moskowitz A, et al. Vasopressors during adult cardiac arrest: a systematic review and meta-analysis. Resuscitation 2019;139:106-21.

6 Soar J, Maconochie I, Wyckoff MH, et al. 2019 international consensus on cardiopulmonary resuscitation and emergency cardiovascular care science with treatment recommendations: summary from the basic life support; advanced life support; pediatric life support; neonatal life support; education, implementation, and teams; and first aid task forces. Circulation 2019;140.

7 Kudenchuk PJ, Brown SP, Daya M, et al. Amiodarone, lidocaine, or placebo in out-of-hospital cardiac arrest. N Engl J Med 2016;374:1711-22.

8 Lind PC, Johannsen CM, Vammen L, et al. Translation from animal studies of novel pharmacological therapies to clinical trials in cardiac arrest: a systematic review. Resuscitation 2021;158:258-69.

9 Lundin A, Djärv T, Engdahl J, et al. Drug therapy in cardiac arrest: a review of the literature. Eur Heart $J$ Cardiovasc Pharmacother 2016;2:54-75.

10 Bramer WM, Giustini D, de Jonge GB, et al. De-duplication of database search results for systematic reviews in endnote. $J$ Med Libr Assoc 2016;104:240-3. 